\title{
Structural Studies Of The Bacteriophage Lambda Holin S105
}

\author{
C. G. Savva*, D. K. Struck**, R. Young** and A. Holzenburg**** \\ * Department of Biology and Microscopy and Imaging Center, Texas A\&M University, College \\ Station, TX 77843 \\ ** Department of Biochemistry and Biophysics, Texas A\&M University, College Station, TX 77843
}

Double-stranded DNA bacteriophages employ a holin-endolysin system to accomplish host lysis (Fig1). Endolysins with a variety of enzyme activities have been reported, including true lysozymes, transglycosylases endopeptidases and amidases all of which act to degrade the peptidoglycan cell wall. To reach their target the endolysin must pass the inner membrane of the host. The holin gene product is thought to create a "hole" or lesion in the inner membrane that allows the endolysin to reach its substrate [1].The bacteriophage $\lambda$ holin S105 is the prototype class I holin comprising 3 alpha-helical transmembrane domains (TMD's). S105 accumulates in the inner membrane and at a specific time, programmed in its sequence, it triggers to form a lesion in the membrane [1].

The structural nature of this lesion or hole remains elusive. To gain insight into the structural features of this small $11 \mathrm{kDa}$ protein we have employed electron microscopy of purified S105 protein. S105-His6 was overexpressed and purified using Ni-NTA chromatography in detergent. Unexpectedly, upon removal of detergent by dialysis, we found S105 to remain in a soluble form. TEM analysis revealed that S105 oligomerized into ring-shaped structures ranging from $20-80 \mathrm{~nm}$ in diameter (Fig.2A). These water soluble oligomers are thought to be double rings in which the amphipathic helices of S105 dimers associate in a way to shield the hydrophobic surfaces of the helices while exposing the hydrophilic surface to the surrounding aqueous medium.

To observe S105 in a more membrane-like environment, the non-anionic detergent dodecyl- $\beta$ maltoside (DDM) was added to the water soluble rings. When examined with TEM, homogenous rings of $23 \mathrm{~nm}$ in diameter were observed (Fig.2B). Furthermore, these rings can form stacks that orient themselves with the ring faces perpendicular to the support film thus providing side-on projections. Single particle analysis of these stacked dimeric rings indicates that the height of these rings is approximately $4 \mathrm{~nm}$ which closely matches the width of a phospholipid bilayer. In addition, the enclosed volume of these rings could house approximatley 40 S105 monomers or the equivalent of $450 \mathrm{kDa}$ of protein per ring (Fig.3). Both the water soluble and detergent solubilized rings demonstrate that S105 is capable of oligomerizing into large supramolecular complexes that resemble the pores formed by many of the water soluble cytolysins with regards to size and overall shape although the cytolysins consist mainly of beta-sheets.

Further biochemical characterizations are being carried out in conjunction with attempts to visualize S105 in native E. coli membranes. These studies suggest that the DDM-solubilized rings might represent a form that is present in the native membrane and ultimately responsible for hole formation.

References

[1] I. N. Wang, D. L. Smith and R. Young, Annu Rev Microbiol. 54 (2000) 799.

[2] S. J. Ludtke, P. R. Baldwin, and W. Chiu, J Struct Biol. 128 (1999) 82. 


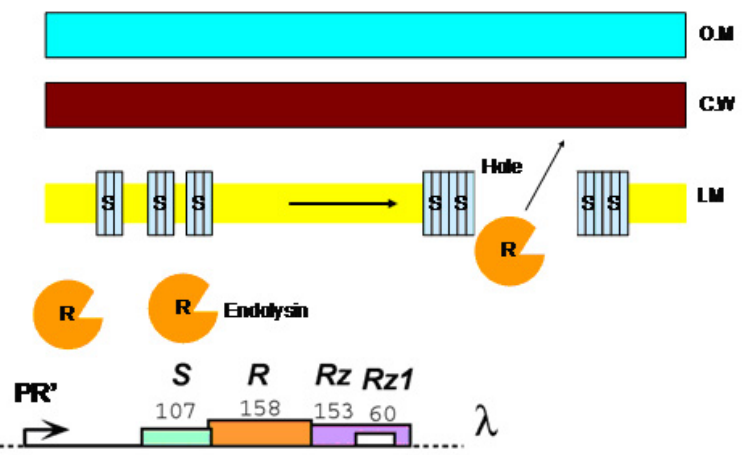

Fig. 1. Cartoon representation of the $\lambda$ lysis system. The lysis cassette is under the control of the native PR' promoter. The RZ and RZ1 gene products are not essential for lysis and have currently no known function.
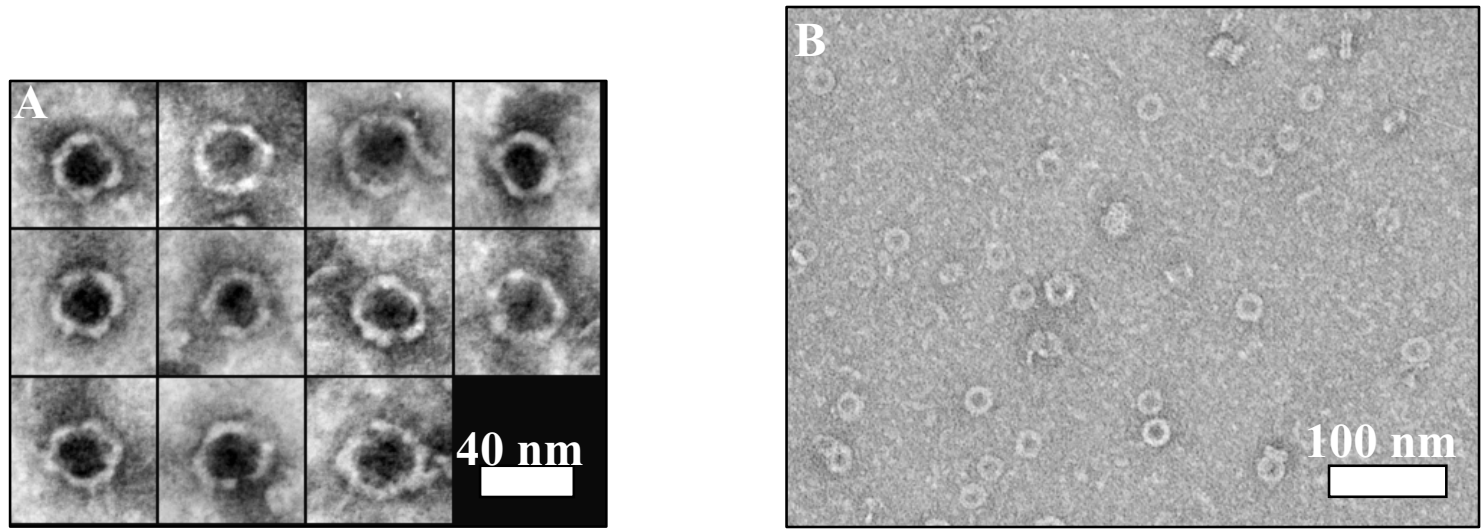

Fig.2. (A) S105 can form water soluble rings in the absence of detergent. (B) S105 rings in the presence of DDM.

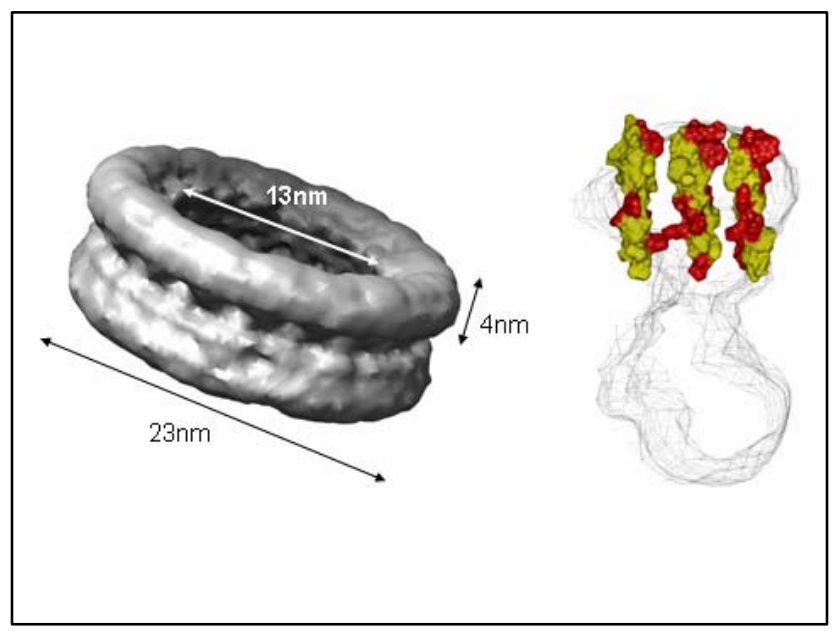

Fig.3. Single particle 3D reconstruction of S105 ring dimers (left) and cross section of a dimer (right) with 3 modeled TMD helices docked to the EM density. The 3D reconstruction was performed using the EMAN software [2]. 